\title{
A MICROSCOPIC THEORY FOR DOMAIN WALL MOTION AND ITS EXPERIMENTAL VERIFICATION IN Fe-AI ALLOY DOMAIN GROWTH KINETICS
}

\author{
J. W. CAHN \\ Institute for Materials Research, National Bureau of Standards, Washington, D.C. 20234, U.S.A. \\ and \\ S. M. ALLEN
}

Dept. of Materials Science and Engineering Massachusetts Institute of Technology Cambridge, Mass. 02139, U.S.A.

\begin{abstract}
Résumé. - Nous proposons une théorie microscopique pour le mouvement des parois de domaine antiphase courbées dans les structures ordonnées et nous trouvons que la vitesse est proportionnelle à la courbure moyenne. Contrairement aux anciens modèles, la vitesse n'est pas proportionnelle à l'énergie libre de la paroi. Les résultats de nos expériences sur la croissance des domaines dans les alliages $\mathrm{Fe}-\mathrm{Al}$ ordonnées pour une gamme de températures, tèmps et compositions sont compatibles avec notre théorie. Tous près de la température critique où l'énergie libre de la paroi tend à zéro, la croissance de domaines n'est pas ralentie.
\end{abstract}

Abstract. - A microscopic theory for curved antiphase domain wall motion in ordered structures leads to a prediction that velocity is proportional to mean curvature. Unlike previous models, the velocity is not proportional to domain wall free energy. Experimental results on domain growth in ordered $\mathrm{FeAl}$ alloys over a range of temperatures, times and compositions, are consistent with the theory. In the vicinity of the critical temperature where domain wall free energy tends to zero, domain growth is not slowed.

Introduction. - Antiphase domain boundaries are a non-equilibrium feature of real ordered alloys. Since there is a positive excess surface free energy associated with these boundaries, they move by diffusion in such a way that the total area of the boundaries is reduced. A long-standing phenomenological theory of interface motion [1-3] states that interfacial velocity is proportional to the thermodynamic driving force, the proportionality constant being a positive quantity called a mobility. The thermodynamic driving force in this theory is the product of the local mean curvature of the boundary and the excess surface free energy per unit area of boundary.

A feature of higher-order transitions in ordering alloys is that the antiphase boundary energy approaches zero as the critical temperature is approached $[4,5]$. Thus, the phenomenological theory of interface motion would seem to indicate that as the critical temperature for ordering is approached, antiphase domain growth should become very sluggish.

In this paper, a new expression for interfacial velocity will be derived using a microscopic model. The excess surface free energy per unit area does not appear in this new formulation, and hence, the behavior of systems near critical points is more clearly represented. Application of the new result to the problem of macroscopic antiphase domain growth leads to a simple experimental test of the theory.

Microscopic theory. - The kinetic equations of continuous ordering reflect the fact that the order parameter $\eta$ is not a conserved quantity. If the free energy is not at a minimum with respect to a local variation in $\eta$ there is an immediate change in $\eta$ given by

$$
-\frac{\partial \eta}{\partial t}=\alpha \frac{\delta F}{\delta \eta}
$$

where $\delta F / \delta \eta$ is the variational derivative of the free energy of the system with respect to a local change in $\eta$, and $\alpha$ is a positive kinetic coefficient. In a non-uniform system [5]

$$
\delta F / \delta \eta=\partial F / \partial \eta-2 \kappa \nabla^{2} \eta
$$

where $\partial F / \partial \eta$ is the rate of change in free energy for uniformly ordered systems and $2 \kappa \nabla^{2} \eta$ is the gradient energy contribution in a system in which $\eta$ is varying spatially. We obtain for the time dependent problem

$$
\frac{\partial \eta}{\partial t}=-\alpha \frac{\partial F}{\partial \eta}+M \nabla^{2} \eta
$$


where $M=2 \alpha \kappa$ has dimensions of a diffusion coefficient $\left(\mathrm{m}^{2} / \mathrm{s}\right)$. This nonlinear equation is similar to the time dependent Ginsburg-Landau equation in which there is no conservation and has been discussed by Kawasaki [6] and Metiu, Kitahari and Ross [7]. Its solution will give the evolution of the $\eta$ field for any conceivable initial $\eta$ distribution. Because of the $\partial F / \partial \eta$ term it is nonlinear.

The equilibrium order parameter $\eta_{e}$ is given by the solution to

$$
\partial F / \partial \eta=0
$$

For systems which have second-order transition $F$ is an even function of $\eta$ and therefore if $\eta_{\mathrm{e}}$ is a solution to (4) so is $-\eta_{\mathrm{e}}$. The antiphase domain wall is the surface between two regions, one with $\eta=\eta_{\mathrm{c}}$ and the other with $\eta=-\eta_{\mathrm{e}}$.

The $\eta$ profile of the equilibrium planar domain wall is given by the solution to

$$
0=\partial F / \partial \eta-2 \kappa \frac{\mathrm{d}^{2} \eta}{\mathrm{d} x^{2}}
$$

subject to the boundary conditions $\eta=\eta_{\mathrm{e}}$ at $x=\infty$ and $\eta=-\eta_{\mathrm{e}}$ at $x=-\infty$. Since the equation is invariant to translation we shall arbitrarily seek the solution $\eta_{\mathrm{p}}(x)$ such that $\eta_{\mathrm{p}}(0)=0$. Surfaces exist only if $\kappa$ is positive.

For a cylindrical or spherically curved domain walls there exists no solution to the equilibrium problem

$$
0=\partial F / \partial \eta-2 \kappa \nabla^{2} \eta
$$

This follows directly from a theorem by Wiener [8] that specifying $\eta$ at a point or line, $r=0$ (instead of on a surface, $r=\infty$ ), will lead to a situation in which no solution to (6) is possible.

We consider the profile $\eta(r, t)$ of a domain wall enclosing a spherical (or cylindrical) domain of radius $R$, where $r$ is a spatial coordinate. Rewriting (3) for cylindrical or spherical symmetry

$$
\partial \eta / \partial t=-\alpha\left(\partial F / \partial \eta-2 \kappa \partial^{2} \eta / \partial r^{2}\right)+M b / r(\partial \eta / \hat{\partial} r)
$$

where $b=1$ or 2 for cylinders or spheres respectively. When $R$ is large compared with the thickness of the interface we can replace $r$ by $R$ in the last term. The equation then has a solution of the form

$$
\eta=\eta_{\mathrm{p}}(r-R)
$$

where $\eta_{\mathrm{p}}$ is the solution to eq. (5) and

$$
\partial R / \partial t=-M b / R .
$$

The boundary moves towards its center of curvature retaining the $\eta_{\mathrm{p}}$ profile without distortion until $R$ becomes comparable to the thickness. Since $b / R$ is the mean curvature $K_{1}+K_{2}$ we may write for the velocity $V$

$$
V=-M\left(K_{1}+K_{2}\right)
$$

Macroscopic theory of domain growth. - Starting with a linear relation between local velocity and mean curvature, eq. (10), and the geometrical relation between the change in area $\delta(\mathrm{d} S)$ when an element $\mathrm{d} S$ of a curved surface moves a distance $V \delta t$ in time $\delta t$

$$
\delta(\mathrm{d} S)=(V \delta t)(\mathrm{d} S)\left(K_{1}+K_{2}\right)
$$

we obtain

$$
\frac{\mathrm{d} S}{\mathrm{~d} t}=-M \int\left(K_{1}+K_{2}\right)^{2} \mathrm{~d} S .
$$

We define the averaged square mean curvature $K^{2}$

$$
K^{2}=\left\langle\left(K_{1}+K_{2}\right)^{2}\right\rangle=\frac{1}{S} \int\left(K_{1}+K_{2}\right)^{2} \mathrm{~d} S .
$$

The surface area in a unit volume of specimen $S_{\mathrm{v}}$ then obeys the relation

$$
\frac{\mathrm{d} S_{\mathrm{v}}}{\mathrm{d} t}=-M K^{2} S_{\mathrm{v}}
$$

If we ignore dislocations the domain structure is multiply connected. As it coarsens both $K^{2}$ and $S_{\mathrm{v}}$ decrease. If we assume that the coarsening structure remains geometrically similar and that only the length scale changes then both $S_{v}^{2}$ and $K^{2}$ are proportional to the reciprocal square of this length scale. Hence

$$
K^{2}=f S_{\mathrm{v}}^{2},
$$

where geometrical similarity implies $f$ is a constant. Substitution for $K^{2}$ and integrating we obtain

$$
\left[S_{v}(t)\right]^{-2}-\left[S_{v}(0)\right]^{-2}=2 f M t
$$

In our experiments $\left[S_{\mathrm{v}}(0)\right]^{-2}$ is negligibly small compared to the other terms even at our shortest aging times.

Experimental results. - Antiphase domain size measurements were made on $\mathrm{Fe}-23$ and -24 atomic percent $\mathrm{Al}$ alloys receiving different ordering treatments. All samples were homogenized and disordered at temperatures above $1050 \mathrm{~K}$ and rapidly quenched to room temperature prior to the ordering treatment. Ordering was carried out in the single-phase $\mathrm{FeAl}$ region of the phase diagram $[9,10]$. Then foils of the ordered material were prepared and examined by transmission electron microscopy. Measurements of antiphase domain boundary surface area per unit volume, $S_{\mathrm{v}}$, were made using standard quantitative analysis techniques [11]. These data are presented in table I and in graphical form in figure 1 where $\log \left[S_{\mathrm{v}}^{2} t\right]^{-1}$ is plotted $v s[T]^{-1} ; t$ and $T$ being the ordering time and temperature, respectively. The data presented in table 1 for the $26.5 \%$ alloy are based on the micrographs of Swann, Duff, and Fisher [12] for an alloy with $\left(\mathrm{DO}_{3}\right)$ order very close to its critical temperature (see discussion). 


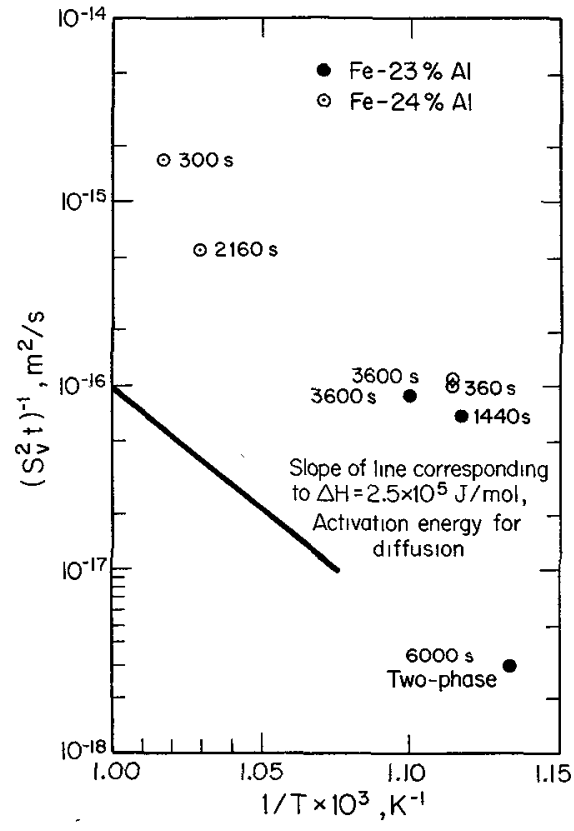

FIG. 1. - Plot of $\log \left[S_{\mathrm{v}}{ }^{2} t\right] v s\left[T^{-1}\right]$ for antiphase domain boundaries in FeAl ordered Fe-A1 alloys. $S_{\mathrm{v}}$ is boundary surface area per unit volume, $t$ is the ordering time, and $T$ is the ordering tempe-

rature. Ordering times are indicated near each datum point.

\section{TABLE I}

Antiphase domain growth data for $\mathrm{Fe}-\mathrm{Al}$ alloys with $\mathrm{FeAl}$ (B2) order

\begin{tabular}{|c|c|c|c|c|c|}
\hline At. $\%$ Al & Temp., $K$ & Time, $s$ & $S_{v}, \mathrm{~m}^{-1}$ & $S_{\mathrm{v}}^{-2}$ & $t^{-1}, \mathrm{~m}^{2} / \mathrm{s}$ \\
\hline $23 \%$ & 909 & 3600 & $1.8 \times 10^{6}$ & 9 & $\times 10^{-17}$ \\
\hline $23 \%$ & 895 & 1440 & $3 \times 10^{6}$ & 7 & $\times 10^{-17}$ \\
\hline $23 \%$ & $883\left(^{a}\right)$ & 6000 & $7 \times 10^{6}$ & 3 & $\times 10^{-18}$ \\
\hline $24 \%$ & 983 & 300 & $1.4 \times 10^{6}$ & 1.7 & $\times 10^{-15}$ \\
\hline $24 \%$ & 972 & 2160 & $9 \times 10^{5}$ & 6 & $\times 10^{-16}$ \\
\hline $24 \%$ & 898 & 3600 & $1.6 \times 10^{6}$ & 1.1 & $\times 10^{-16}$ \\
\hline $24 \%$ & 898 & 360 & $5 \times 10^{6}$ & 1 & $\times 10^{-16}$ \\
\hline $26.5 \%$ & $823\left(^{b}\right)$ & 100,800 & $\times 10^{6}$ & 2 & $\times 10^{-18}$ \\
\hline
\end{tabular}

(a) May have $\alpha$-phase coating domain wall.

(b) $\mathrm{DO}_{3}$ order from Ref. [12]

Discussion. - The microscopic theory leads directly to a linear relation between mean curvature and boundary velocity. It also demonstrates that, until the radius of curvature becomes comparable to domain wall thickness, the profile of a moving curved wall is not too different from that of an equilibrium planar wall.

Thermodynamics requires that surfaces move in such a way as to reduce total surface free energy. Since the specific surface free energy is positive and constant, this is synonymous with area reduction. Surfaces must move toward the center of curvature of the larger principal curvature. Since $M$ is positive, the linear law is consistent with thermodynamics.
Since the surface free energy tends to zero at the critical point, the driving force tends to zero also. It is therefore surprising that $M$ as derived herein remains finite. The two factors that determine $M$ are the rate constant $\alpha$ and the gradient energy coefficient $\kappa$. The function $F$ which is an important factor in surface free energy and surface structure and contributes to the non-linear behavior of the kinetic eq. (3) cancels out. The quantities $\eta_{\mathrm{e}}$ (which relates to the number of atoms that must move) and the thickness of the wall also do not appear.

In figure 1 and table I we report our data to test this theory and the macroscopic theory. The two alloys have widely different critical temperatures. $S_{\mathrm{v}}^{2} t$ seems to be a function of temperature alone indicating both that the linear dependence of velocity on curvature and the geometrical similitude are consistent with the data. As indicated in figure 1 the temperature dependence of $f M$ follows that of the diffusion coefficient (13). There is no marked deviation as either alloy approaches its critical temperature.

The experimental temperature range for the $23 \%$ alloy is bounded by the critical temperature at $902 \pm 3 \mathrm{~K}$ and the two-phase region which begins at $885 \pm 3 \mathrm{~K}$. The one low point in figure 1 is presumably for a two-phase alloy. Because a small amount of $\alpha$ would uniformly coat the domain wall, it is difficult to detect microscopically. The critical temperature for our $24 \%$ alloy is above $986 \mathrm{~K}$ and well above the published temperature $[9,14,15]$.

Swann, Duff, Fisher [12] studied the vicinity of the critical temperature in a $\mathrm{DO}_{3}$ alloy kept in a temperature gradient for $28 \mathrm{hrs}$. Their published micrographs indicate no change to smaller domain size even at the point within $1 \mathrm{~K}$ of the critical temperature. They span an order of magnitude in undercooling and thus, according to theory, about a factor of twenty in surface free energy. Their domain size falls approximately on a continuation of our figure 1 even though it is a different kind of domain wall.

Factors such as critical opalescence and adsorption because of the vicinity of the tricritical point seem to play no important role in altering the boundary velocity.

We conclude that the kinetic theory leads to a simple result for domain wall motion which is consistent with domain growth observations near the critical temperature. Any theory which implies that the temperature dependence of this velocity tends to zero at the critical temperature along with the surface free energy is not consistent with either our theory or data.

Acknowledgments. - We are grateful to the National Science Foundation for support of the portion of the research performed at MIT. We are indebted to $\mathrm{W}$. Boettinger for a critical review of the manuscript. 


\section{References}

[1] Smoluchowski, R., Phys. Rev. 83 (1951) 69.

[2] Turnbull, D., Trans. AIME 191 (1951) 661.

[3] Mullins, W. W., J. Appl. Phys. 27 (1956) 900.

[4] OnSAGER, L., Phys. Rev. 65 (1944) 117.

[5] Kikuchi, R. and CaHn, J. W., J. Phys. Chem. Solids 23 (1962) 137.

[6] Kawasaki, K., Prog. Theor. Phys. 57 (1977) 410.

[7] Metiu, H., Kitahari, K. and Ross, J., J. Chem. Phys. 64 (1976) 292.

[8] Courant, R. and Hirbert, D., Methods of Mathematical Physics, Volume II (Interscience N.Y.) (1962) 305-6. We are indebted to F. J. Almgren for calling our attention to this theorem.
[9] Swann, P. R., Duff, W. R. and Fisher, R. M., Trans. TMSAIME 245 (1969) 851.

[10] Allen, S. M. and CaHn, J. W., Acta Met. 23 (1975) 1017.

[11] Smith, C. S. and Guttman, L., Trans. AIME 197 (1953) 81.

[12] Swann, P. R., Duff, W. R. and Fisher, R. M., Phys. Status Solidi 37 (1970) 577.

[13] Nishida, K., Yamamoto, T. and Nagata, T., Trans. Japan Inst. Metals 12 (1971) 310.

[14] Swann, P. R., Durf, W. R. and Fisher, R. M., Met. Trans. 3 (1972) 409.

[15] Окамото, P. R. and Веск, P. A., Met. Trans. 2 (1971) 569. 